\title{
When group dispersal and Allee effect shape metapopulation dynamics
}

\section{Soubeyrand, Samuel}

2017-04

Soubeyrand, S \& Laine , A-L 2017 , ' When group dispersal and Allee effect shape

metapopulation dynamics ' , Annales Zoologici Fennici , vol. 54 , no. 1-4 , pp. 123-138 . https://doi.org/10.5735/086.0

http://hdl.handle.net/10138/225735

https://doi.org/10.5735/086.054.0112

cc_by

publishedVersion

Downloaded from Helda, University of Helsinki institutional repository.

This is an electronic reprint of the original article.

This reprint may differ from the original in pagination and typographic detail.

Please cite the original version. 


\title{
When group dispersal and Allee effect shape metapopulation dynamics
}

\author{
Samuel Soubeyrand ${ }^{1, *} \&$ Anna-Liisa Laine ${ }^{2}$ \\ 1) BioSP, INRA, FR-84914 Avignon, France ("corresponding author's e-mail: samuel.soubeyrand@ \\ inra.fr) \\ 2) Metapopulation Research Centre, Department of Biosciences, P.O. Box 65, FI-00014 University \\ of Helsinki, Finland
}

Received 15 Nov. 2016, final version received 16 Feb. 2017, accepted 17 Feb. 2017

Soubeyrand, S. \& Laine, A.-L. 2017: When group dispersal and Allee effect shape metapopulation dynamics. - Ann. Zool. Fennici 54: 123-138.

The dispersal ability of a species will be critical for how population dynamics are realized in spatially structured systems. To date, the effect of group dispersal on metapopulation dynamics is poorly understood. Here, we investigate how group dispersal and Allee effects shape metapopulation dynamics identifying conditions in which group dispersal can be an advantage over independent dispersal. We approach this question by building and analysing a Markovian random walk for metapopulation dynamics including group dispersal and Allee effect. This Markovian random walk is analogous to the discrete-time Stochastic Patch Occupancy Model (SPOM). We find that intermediate group sizes may lead to larger and more sustainable metapopulations in the presence of an Allee effect. Hence, understanding how group size variation and realized (meta)population dynamics are linked offers an exciting future venue for research that is expected to yield key insights into the ecology and evolution of populations occupying spatially structured environments.

\section{Introduction}

Metapopulations are an assembly of spatially delimited local populations that are coupled by some degree of migration (Hanski \& Gaggiotti 2004). Classic metapopulation dynamics (Levins 1969, 1970) focus on the processes of local extinction and re-colonization of local habitat patches in the same manner as population dynamics are concerned with births and deaths of individuals. Since their development, metapopulation models have evolved from being spatially implicit to being spatially explicit with broad real-world applicability, for example in conservation biology (Hanski 1999). Indeed, the metapopulation theory is no longer an academic debate as a broad range of species have been shown to persist regionally via metapopulation dynamics, e.g. butterflies (Hanski 1999), plants (Ouborg 1993), frogs (Carlson \& Edenhamn 2000) and owls (Lahaye et al. 1994). Importantly, metapopulation theory has also captured well the epidemiological dynamics of many diseases, as reviewed in Grenfell and Harwood (1997) and Parratt et al. (2016).

Patchy distribution of suitable habitat either naturally or as a result of human induced habitat fragmentation - has been considered a defining element of metapopulations. However, the dispersal ability of a species will be critical 
for how population dynamics are realized in spatially structured systems (Hanski 1999). The dispersal process may be divided into three phases: departure, movement and settlement (Matthysen 2012). Following departure, the movement process is often described by the "basic" dispersal kernel, which is based on the distribution of dispersed units. Notably, this basic dispersal kernel frequently deviates from the realized distribution of individuals in the field due to spatial variation in settlement success [i.e., the "effective" dispersal kernel, which is based on the distribution of successfully dispersed units; Nathan et al. (2012)]. To date, the question of why to disperse has been extensively investigated from an evolutionary perspective, and these investigations have generally established a significant advantage associated with dispersal ability (Travis \& Dytham 2002, Ronce 2007, Rousset 2012, Starrfelt \& Kokko 2012). Beyond this first question, the amazing diversity of dispersal mechanisms that animals, plants, fungi, peat mosses and other organisms have developed leads to the second question of how to disperse. In addition to the many modes (e.g. active $v s$. passive) of individuals' movement through space, both individual and group dispersal have been documented in a wide range of species. Indeed, it may be that in many systems, whether it is individuals or groups of individuals dispersing may play a significant role in the settlement phase of dispersal, thereby creating discrepancy between the basic and realized dispersal kernels. The costs of solitary emigration implied by Allee effects - for example when mates become rare and opportunities for reproduction are limited (Courchamp et al. 1999) - may be overcome by colonization of habitats by groups of individuals, thereby increasing the likelihood of successful establishment following arrival at a previously uncolonized yet suitable patch in a metapopulation.

Group dispersal is common in social insects (colony fission or budding in ants and bees), vertebrates (e.g. lions) and collective swarming in bacteria (Clobert et al. 2012). Pathogen spores are known to form clumps (Ferrandino \& Aylor 1987), suggesting that the size of the dispersing unit may have profound consequences for realized epidemiological dynamics (Tack et al. 2014). Group dispersal may be an active process or a more passive process promoted by external factors, such as extreme weather events which are recognized to be a powerful mechanism for moving aggregates of individuals from one site to another (Aylor 1990). However, only a few modelling approaches characterizing this type of dispersal and its selective advantages exist in the literature. Meerson and Ovaskainen (2013) proposed a modelling framework for representing immigration-extinction dynamics in which group dispersal can be included. Using this framework, Ovaskainen (2017) showed that the colonization rate is maximized with intermediate group sizes in the presence of an Allee effect. In parallel, Soubeyrand et al. (2015) showed, using an evolutionary model embedded in a demographic model and simulation experiments, how the spatial limits and fragmentation of the species habitat shape the frequencies of the independent and group dispersal strategies. In this article, we describe how group dispersal and Allee effects may shape metapopulation dynamics by identifying conditions in which group dispersal can present an advantage over independent dispersal. We focus on two key metapopulation parameters: metapopulation size and time to extinction. Specifically, we ask: (1) How to include group dispersal in a metapopulation model whose behaviour can be analytically investigated, to be able to draw general conclusions? (2) Do group dispersal and Allee effect have a monotonic influence on metapopulation dynamics or is there a trade-off between the two? (3) How do group dispersal and Allee effect influence the stability of a metapopulation?

\section{Two-population system}

Here, we consider a basic system with a single source population affected by a dispersal event, which potentially generates a new population. This simple system is defined and analysed below and is used as a basis for the metapopulation model.

\section{Source population}

Consider a source population uniformly spread 
within a disk whose center is $x_{\mathrm{s}}$ in the planar space $\mathbb{R}^{2}$ at the altitude $0 \mathrm{~m}$ (i.e. the ground level). Let $\lambda_{0}$ denote the initial intensity of this population (the intensity is measured, for instance, by a number of individuals per square meter). Without loss of generality, this intensity is set to $1\left(\lambda_{0}=1\right)$.

Suppose that the source population is simultaneously affected by the two following processes (because of an extreme weather event for example): (1) migration of a fraction $\lambda_{\mathrm{m}}$ of the population, and (2) destruction of a fraction $\lambda_{d}$ of the population, with $\lambda_{\mathrm{m}}+\lambda_{\mathrm{d}} \leq \lambda_{0}=1$.

Right after the migration/destruction event, the intensity of the source population is still constant but equal to $\lambda_{0}-\lambda_{\mathrm{m}}-\lambda_{\mathrm{d}}=1-\lambda_{\mathrm{m}}-\lambda_{\mathrm{d}}$.

\section{Group dispersal}

The migrating fraction $\lambda_{\mathrm{m}}$ of the source population is dispersed as a single group. Indeed, the migrating fraction is assumed to be released within a short duration and, consequently, to face approximately constant release and transport conditions. However, as in Soubeyrand et al. (2011, 2014), the group is assumed to be diffused during the transport: the air volume containing the migrating fraction of the source population is in expansion. This feature is depicted in Fig. 1 and is modelled below.

When the migrating fraction reaches the atmosphere layer, at altitude $h$, where it will be transported horizontally, it is spread horizontally like a Gaussian kernel centred around the source population centre $x_{\mathrm{s}}$ and with variance $\sigma_{\text {up }}^{2}$. Therefore, at this stage, the intensity $\lambda_{\text {up }}$ of the migrating fraction orthogonally projected to the planar space satisfies for all $x \in \mathbb{R}^{2}$ :

$$
\lambda_{\text {up }}(x)=\frac{\lambda_{\text {m }}}{2 \pi \sigma_{\text {up }}^{2}} \exp \left(-\frac{\| x-\left.x_{\text {s }}\right|^{2}}{2 \sigma_{\text {up }}^{2}}\right),
$$

where $\left\|x-x_{\mathrm{s}}\right\|$ is the Euclidean distance between $x$ and $x_{\mathrm{s}}$ in $\mathbb{R}^{2}$.

Then, the migrating fraction is transported horizontally to a colonization location $x_{\mathrm{c}}$. During this horizontal move, the migrating fraction continues to be diffused and the strength of the diffusion depends on the distance travelled, namely $\left\|x_{\mathrm{c}}-x_{\mathrm{s}}\right\|$. Finally, the migrating fraction is depos-

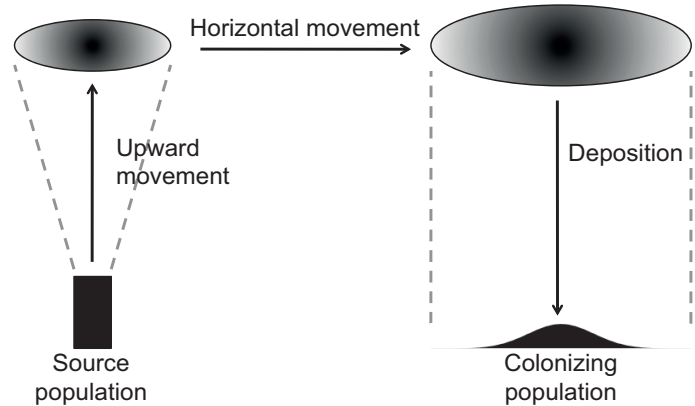

Fig. 1. Transport and diffusion of the migrating fraction of the source population.

ited to the ground without additional diffusion. Therefore, after the deposition, the intensity $\lambda_{c}$ of the migrating fraction over the planar space is proportional to a Gaussian kernel centred around the colonization location $x_{\mathrm{c}}$ and with variance $\sigma_{\text {up }}^{2}+v\left\|x_{\mathrm{c}}-x_{\mathrm{s}}\right\|^{\eta}$. Thus, for all $x \in \mathbb{R}^{2}$,

$$
\begin{aligned}
\lambda_{\mathrm{c}}(x) & =\frac{\lambda_{\mathrm{m}}}{2 \pi\left(\sigma_{\text {up }}^{2}-v\left\|x_{\mathrm{c}}-x_{\mathrm{s}}\right\|^{\eta}\right)} \\
& \exp \left(-\frac{\left\|x-x_{\mathrm{c}}\right\|^{2}}{2\left(\sigma_{\text {up }}^{2}+v\left\|x_{\mathrm{c}}-x_{\mathrm{s}}\right\|^{p}\right)}\right)
\end{aligned}
$$

where $v$ and $\eta$ are positive parameters modulating the diffusion during the horizontal move of the migrating fraction. According to Eq. 2, the minimal diffusion is determined by $\sigma_{\text {up }}^{2}$ and the diffusion increases with the travelled distance $\left\|x_{c}-x_{s}\right\|$, up to the scaling factor $v$ and the shape parameter $\eta$. The greater $v$, the greater the effect of the travelled distance on the diffusion. In addition, when $\eta$ is smaller (or greater) than 1, consecutive increments in the travelled distance have decreasing (or increasing) effects on the diffusion.

It has to be noted that the choice of the Gaussian kernels used above is rather arbitrary, even if it could be justified theoretically by considering that the migrating fraction consists of a large number of particles following independent Brownian motions stopped at a fixed time depending on the travelled distance (Soubeyrand et al. 2011). However, if we suppose that an extreme weather event generates migration, as suggested above, then the Gaussian assumption might be strongly unrealistic. In addition, the diffusion and the travelled distance $\left\|x_{c}-x_{\mathrm{s}}\right\|$ could 
depend on the duration of the extreme weather event. Another desirable feature for $\lambda_{c}$ could be that its support is finite (to account for finite distance movements only). Moreover, the dispersal parameters in Eqs. 1 and 2 could depend on group size, for behavioural reasons in the case of active dispersers or bio-physical reasons in the case of passive particles (e.g. when particles are sticky or clumped; Soubeyrand et al. (2015) considered such a dependence between dispersal parameters and group size). In the framework that we propose, more complicated spatio-temporal scenarios could be easily taken into account: the form of $\lambda_{c}$ could be replaced by more elaborated or finite-support functions, and the subsequent equations (which essentially require the knowledge of the maximum intensity of the colonizing population) could be modified accordingly.

\section{Survival and emergence}

After the migration/destruction event, the question of the survival of the source population and the question of the emergence of the colonizing population arise. An Allee effect is assumed to operate as follows:

- If the new intensity $1-\lambda_{\mathrm{m}}-\lambda_{\mathrm{d}}$ of the source population is above the threshold $\lambda_{\text {min }}$, then the source population survives and returns to its initial state, that is to say the unit level; otherwise, it becomes extinct.

- If the maximum intensity of the colonizing population, namely $\lambda_{c}\left(x_{c}\right)$, is above the threshold $\lambda_{\text {min }}$, then the colonizing population emerges and reaches the unit level over a disk with centre $x_{\mathrm{c}}$ (like the source population at its initial state); otherwise, it becomes extinct.

Thus, there are four possible outputs after the migration/destruction event:

1. The source population (SP) survives and the colonizing population (CP) emerges if:

$$
\begin{cases}1-\lambda_{\mathrm{m}}-\lambda_{\mathrm{d}} & \geq \lambda_{\text {min }} \\ \lambda_{\mathrm{m}} /\left\{2 \pi\left(\sigma_{\text {up }}^{2}+v r^{\eta}\right)\right\} & \geq \lambda_{\text {min }}\end{cases}
$$

where $r=\left\|x_{c}-x_{\mathrm{s}}\right\|$ is the distance between the centers of the source and colonizing populations;

2. SP survives and $\mathrm{CP}$ goes extinct if:

$$
\begin{cases}1-\lambda_{\mathrm{m}}-\lambda_{\mathrm{d}} & \geq \lambda_{\min } \\ \lambda_{\mathrm{m}} /\left\{2 \pi\left(\sigma_{\text {up }}^{2}+v r^{\eta}\right)\right\} & <\lambda_{\text {min }},\end{cases}
$$

3. SP goes extinct and $\mathrm{CP}$ emerges if:

$$
\begin{cases}1-\lambda_{\mathrm{m}}-\lambda_{\mathrm{d}} & <\lambda_{\text {min }} \\ \lambda_{\mathrm{m}} /\left\{2 \pi\left(\sigma_{\text {up }}^{2}+v r^{\eta}\right)\right\} & \geq \lambda_{\text {min }},\end{cases}
$$

4. SP and CP go extinct if:

$$
\begin{cases}1-\lambda_{\mathrm{m}}-\lambda_{\mathrm{d}} & <\lambda_{\text {min }} \\ \lambda_{\mathrm{m}} /\left\{2 \pi\left(\sigma_{\mathrm{up}}^{2}+v r^{\eta}\right)\right\} & <\lambda_{\min }\end{cases}
$$

Figure 2 presents examples of phase diagrams describing the outputs of the two-population system. If $\lambda_{\mathrm{m}}, \lambda_{\mathrm{d}}$ and $\lambda_{\min }$ are all different from 0 and 1 , each of the four outputs can be reached by the system, depending on the values of $\sigma_{\text {up }}^{2}, v, r$ and $\eta$ ) (see Fig. 2a and b). When the diffusion of the migrating fraction is large (i.e., large $\sigma_{\text {up }}^{2}$ or large $v r^{\eta}$ ), at least one of the two populations goes extinct: either the migrating fraction is small, SP survives and CP vanishes because of the diffusion, or the migrating fraction is large, $\mathrm{SP}$ vanishes and $\mathrm{CP}$ emerges despite the diffusion (see Fig. 2d and g). When the diffusion of the migrating fraction is even larger, the colonizing population cannot emerge (see Fig. $2 \mathrm{e}$ and $\mathrm{h}$ ). Curves in Fig. $2 \mathrm{f}$ and $\mathrm{g}$ were drawn for a value of the power $\eta>1$; such a value tends to increase the diffusion of the colonizing population at large distances $(r>1)$ and to reduce the possibility that the colonizing population emerges. If $\eta<1$, the curves are no more concave but convex (see Fig. 3).

To summarize, in our modelling framework dispersal in groups is a two-edged sword: it can permit a migrating population to be able to surmount the Allee threshold, but it also might pose a risk in the source population, by dropping its intensity below that threshold. 

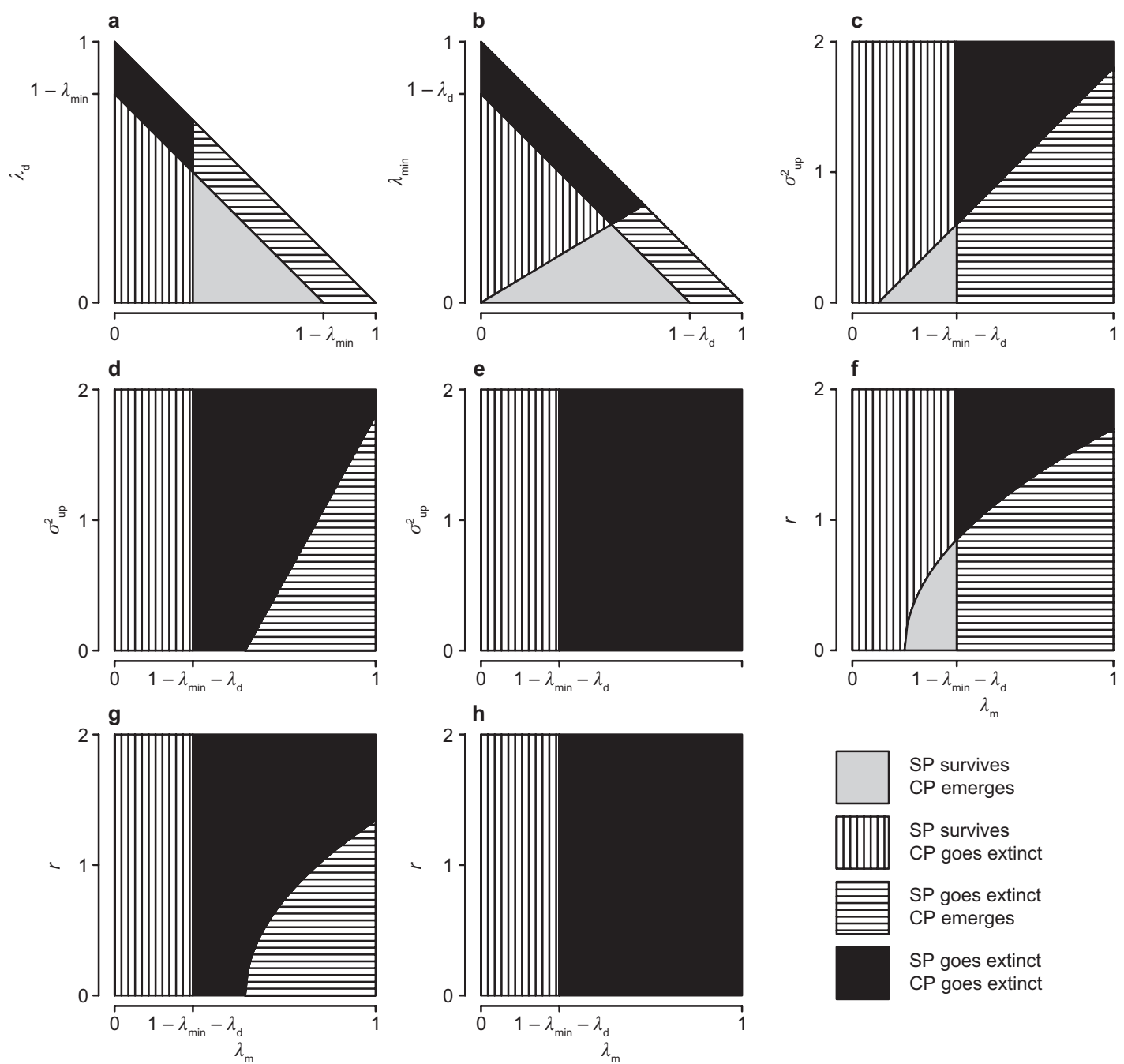

SP survives $\mathrm{CP}$ emerges

SP survives CP goes extinct

SP goes extinct $\mathrm{CP}$ emerges

SP goes extinct CP goes extinct

Fig. 2. Phase diagrams describing the outputs of the two-population system. Plots were drawn with the following parameter values: $(\mathbf{a})\left(\lambda_{\text {min }}, \sigma_{\text {up }}^{2}+v r^{\eta}\right)=(0.2,3 / 4 \pi)$, (b) $\left(\lambda_{\mathrm{d}}, \sigma_{\text {up }}^{2}+v r^{\eta}\right)=(0.2,5 / 6 \pi),(\mathbf{c})\left(\lambda_{\mathrm{d}}, \lambda_{\text {min }}, v r^{\eta}\right)=(0.4-(1 / 2 \pi)$, $1 / 2 \pi, 0.1)$, (d) $\left(\lambda_{\mathrm{d}}, \lambda_{\text {min }}, v r^{\eta}\right)=(0.3-(1 / 3.6 \pi), 1 / 3.6 \pi, 0.9),(\mathbf{e})\left(\lambda_{\mathrm{d}}, \lambda_{\min }\right)=(0.3-(1 / 3.6 \pi), 1 / 3.6 \pi)$ and $v r^{\eta}>1.8,(\mathbf{f})\left(\lambda_{\mathrm{d}}\right.$, $\left.\lambda_{\text {min }}, \sigma_{\text {up }}^{2}, v, \eta\right)=(0.4-(1 / 2 \pi), 1 / 2 \pi, 0.2,10 / 9,2),(\mathbf{g})\left(\lambda_{\mathrm{d}}, \lambda_{\min }, \sigma_{\text {up }}^{2}, v, \eta\right)=(0.3-(1 / 2 \pi), 1 / 2 \pi, 0.5,10 / 9,2)$; and (h) $\lambda_{\mathrm{d}}+$ $\lambda_{\min }=0.3, \sigma_{\text {up }}^{2}>1, v>0$ and $\eta>0$.

\section{Metapopulation dynamics}

The objectives here are (i) to generalize the twopopulation system into a metapopulation system, i.e., a dynamic system with the number of populations that varies in the set $\mathbb{N}$ of non-negative integers, and (ii) to study the properties of this dynamic system. Here, space is not explicitly represented to be able to obtain closed mathematical forms for the transition probabilities.

\section{Specification of model components}

In the metapopulation model defined below, the migrating and destroyed fractions as well as the dispersal distances are random variables. This specification leads to a metapopulation whose size follows a Markovian random walk.

\section{Migration/destruction events}

Such events occur at times $t_{1}, t_{2}, \ldots$, and each of 

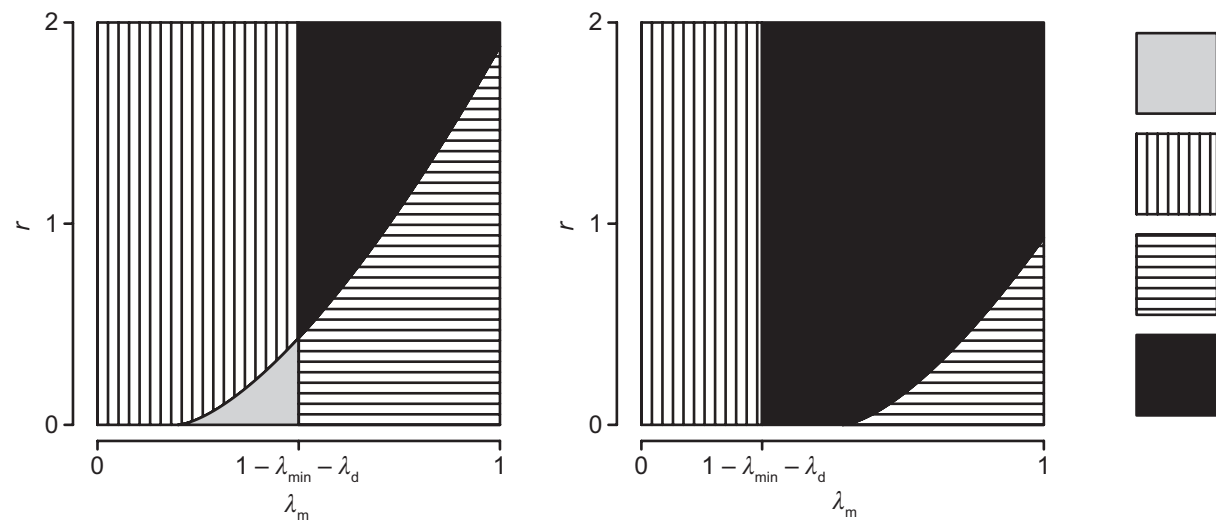

SP survives

CP emerges

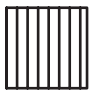

SP survives

CP goes extinct

Fig. 3. Phase diagrams describing the outputs of the two-population system. Plots were drawn with the following parameter values. Left-hand-side panel: $\left(\lambda_{\mathrm{d}}, \lambda_{\min }, \sigma_{\mathrm{up}}^{2}, v, \eta\right)=(0.5-(1 / 2 \pi), 1 / 2 \pi, 0.2,5 / 6,2 / 3)$; right-hand-side panel: $\left(\lambda_{\mathrm{d}}, \lambda_{\min }, \sigma_{\text {up }}^{2}, v, \eta\right)=(0.3-(1 / 2 \pi), 1 / 2 \pi, 0.5,5 / 6,2 / 3)$.

these events affects only one population, i.e., one source population (SP). SP at time $t_{i}$ is uniformly drawn from among all the present populations and the draw is conditionally independent from the previous draws at time $t_{1}, \ldots, t_{i-1}$, given the state of the system between $t_{i-1}$ and $t_{i}$.

\section{Migrating and destroyed fractions}

The migrating fraction $\lambda_{\mathrm{m}}$ and the destroyed fraction $\lambda_{\mathrm{d}}$ are two proportions whose sum is greater than or equal to 1 . The fractions $\lambda_{\mathrm{m}}$ and $\lambda_{\mathrm{d}}$ are independently drawn at times $\left\{t_{1}, t_{2}, \ldots\right\}$ in uniform distributions in $\left[0, \alpha_{\mathrm{m}}\right]$ and $\left[0, \alpha_{\mathrm{d}}\right]$, respectively, where $0 \leq \alpha_{\mathrm{m}}+\alpha_{\mathrm{d}} \leq 1$. Thus, the probability density function and the expectation of $\left(\lambda_{\mathrm{m}}, \lambda_{\mathrm{d}}\right)$ are:

$$
f\left(\lambda_{\mathrm{m}}, \lambda_{\mathrm{d}}\right)=\frac{1}{\alpha_{\mathrm{m}}} \mathbb{1}\left(0 \leq \lambda_{\mathrm{m}} \leq \alpha_{\mathrm{m}}\right) \frac{1}{\alpha_{\mathrm{d}}} \mathbb{1}\left(0 \leq \lambda_{\mathrm{d}} \leq \alpha_{\mathrm{d}}\right)
$$

and

$$
\mathbb{E}\left(\lambda_{\mathrm{m}}, \lambda_{\mathrm{d}}\right)=\left(\frac{1}{2 \alpha_{\mathrm{m}}}, \frac{1}{2 \alpha_{\mathrm{d}}}\right),
$$

where $\mathbb{1}(\cdot)$ is the indicator function $(\mathbb{1}(E)=1$ if event $E$ occurs, otherwise $1(E)=0)$. A new pair of proportions $\left(\lambda_{\mathrm{m}}, \lambda_{\mathrm{d}}\right)$ is drawn for each migration/destruction event and this draw is independent from the previous ones.

Here, we use a rather simple model for drawing $\left(\lambda_{\mathrm{m}}, \lambda_{\mathrm{d}}\right)$. This model allows us to obtain closed mathematical formulas for analysing the metapopulation dynamics; see below. Alternative and finer modelling could be based, for example, on the Dirichlet distribution.

\section{Dispersal distances}

Migrating fractions are assumed to be dispersed at random distances $\left\|x_{\mathrm{c}}-x_{\mathrm{s}}\right\|$ independently drawn at times $\left\{t_{1}, t_{2}, \ldots\right\}$ under an exponentialpower distribution (defined over $\mathbb{R}_{+}$) with parameters $\beta>0$ and $\gamma>0$. Thus, the probability density function and the expectation of $\left\|x_{\mathrm{c}}-x_{\mathrm{s}}\right\|$ are:

$$
g\left(\left\|x_{\mathrm{c}}-x_{\mathrm{s}}\right\|\right)=\frac{\gamma}{\beta^{1 / \gamma} \Gamma(1 / \gamma)} \exp \left(-\frac{\left\|x_{\mathrm{c}}-x_{\mathrm{s}}\right\|^{\gamma}}{\beta}\right)
$$

and

$$
\mathbb{E}\left(\left\|x_{\mathrm{c}}-x_{\mathrm{s}}\right\|\right)=\frac{\beta^{1 / \gamma} \Gamma(2 / \gamma)}{\Gamma(1 / \gamma)} .
$$

For $\gamma<1$, the dispersal function is fat-tailed.

Colonizing populations are assumed to not overlap with existing populations. This assumption is defensible if the spatial extent of each population is small with respect to the mean dispersal distance and the number of populations is not too large. Hence, we will assume that the maximal number of populations is $N^{\max }<\infty$. It has to be noted that a spatially explicit version of the model could circumvent this assumption, but such a model would not allow us to obtain analytical results. More generally, exploring spatially explicit scenarios would be interesting to 
numerically assess (i) under which conditions our temporal-only model makes adequate prediction and (ii) which spatial features and processes lead to deviations between our model and a spatially-explicit model.

\section{Regeneration/emergence of populations}

After each migration/destruction event, if the source population survives (see survival rules in the section 'Survival and emergence'), it reaches the unit level. Similarly, if the colonizing population emerges (see emergence rules in the section 'Survival and emergence'), it reaches the unit level.

\section{Additional assumptions for the metapopulation}

Between the migration/destruction events modelled above, we assume that minor migrations between existing populations occur but the modification in the population intensities are temporary (i.e., the intensity of each population can be assumed to remain equal to the unit level). In addition, we assume that between the migration/ destruction events no existing population vanishes and no new population emerges.

\section{Dynamics of the metapopulation size}

Under the assumptions made above, the metapopulation size (i.e. the number of populations) follows a Markovian random walk over the set of non-negative integers $\mathbb{N}$. In this random walk, the state "zero" (i.e. the metapopulation extinction) is an absorbing state and the possible transitions are only increments $+1 .-1$ and +0 . Let $N_{i}$ denote the metapopulation size after the migration/destruction event occurring at time $t_{i}$. The transition probabilities of the random walk are the same for any $N_{i}$ in $\left\{1, \ldots, N^{\max }-1\right\}$ but are specific for $N_{i}=0$ and $N_{i}=N^{\max }$ :

- When $1 \leq N_{i}<N^{\max }$,

$$
\mathbb{P}\left(N_{i+1}=N_{i}+1\right)=p_{+1},
$$

$$
\begin{aligned}
& \mathbb{P}\left(N_{i+1}=N_{i}-1\right)=p_{-1}, \\
& \mathbb{P}\left(N_{i+1}=N_{i}\right)=1-p_{+1}-p_{-1}=p_{+0},
\end{aligned}
$$

where $p_{+1}$ and $p_{-1}$ are provided in Eqs. A2 and A3 in Appendix.

- When $N_{i}=0$,

$$
\begin{aligned}
& \mathbb{P}\left(N_{i+1}=N_{i}+1\right)=0, \\
& \mathbb{P}\left(N_{i+1}=N_{i}-1\right)=0, \\
& \mathbb{P}\left(N_{i+1}=N_{i}\right)=1 .
\end{aligned}
$$

- When $N_{i}=N^{\max }$,

$$
\begin{aligned}
& \mathbb{P}\left(N_{i+1}=N_{i}+1\right)=0, \\
& \mathbb{P}\left(N_{i+1}=N_{i}\right)=p_{+0}+p_{+1}, \\
& \mathbb{P}\left(N_{i+1}=N_{i}-1\right)=p_{-1} .
\end{aligned}
$$

This Markovian random walk can be viewed, in the context of metapopulation dynamics, as a discrete-time Stochastic Patch Occupancy Model (SPOM; Ovaskainen \& Hanski 2004a), where the time coincides with the indices $i \in\{1,2, \ldots\}$ of the migration/destruction events occurring at the real times $\left\{t_{1}, t_{2}, \ldots\right\}$.

\section{Quasi-stationary distribution and extinction time}

In general, after a sufficiently long time, any metapopulation governed by the transition probabilities provided above vanishes. There is one exception to this statement: in the specific case $\alpha_{\mathrm{m}}+\alpha_{\mathrm{d}}+\lambda_{\min } \leq 1$, the metapopulation cannot vanish because the intensity of any SP remains above the threshold $\lambda_{\text {min }}$ even if the migrating and destroyed fractions $\lambda_{\mathrm{m}}$ and $\lambda_{\mathrm{d}}$ are equal to their maximum values $\alpha_{\mathrm{m}}$ and $\alpha_{\mathrm{d}}$, respectively.

Because the destiny of a metapopulation is, in general and in theory, to go extinct, we need tools to describe the behaviour of the metapopulation before extinction. Thus, following (Ovaskainen \& Hanski 2004b: box 4.2), we analysed our metapopulation dynamics including group dispersal and Allee effect with the following tools:

- the quasi-stationary distribution of the metapopulation size, which gives the conditional probability distribution for the size of the 


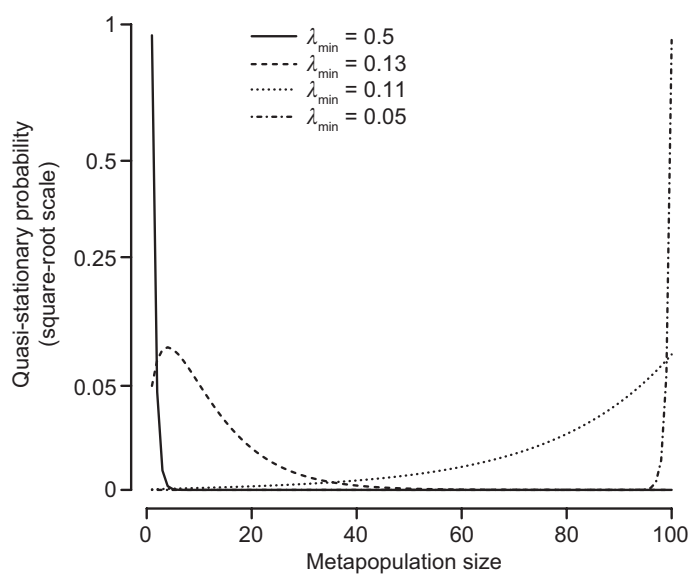

Fig. 4. Quasi-distribution of the metapopulation size for different values of the threshold $\lambda_{\min }$. The other parameters are fixed to $\alpha_{\mathrm{m}}=0.5, \alpha_{\mathrm{d}}=0.5, \sigma_{\text {up }}^{2}=0.1, v=0.1$, $\eta=1, \beta=100, \gamma=1$, and $N^{\max }=100$.

metapopulation given that the metapopulation has not reached extinction;

- the expected time to extinction, which gives, for each initial size of the metapopulation, the average number of migration/destruction events that leads to the extinction of the metapopulation.

The exact definitions of these tools and the corresponding formula are provided in Darroch \& Seneta (1967: section 4) and Ovaskainen and Hanski (2004b: box 4.2). These formulas, which require the transition probabilities provided in the previous subsection as input, were used to produce the plots shown in the Results. Note that the quasi-stationary distribution that we considered here does not depend on the initial size of the metapopulation. Thus, as explained by Darroch and Seneta (1967), such a quasi-stationary distribution is relevant for the realizations in which the time to extinction is long.

\section{Results}

Regarding the strength of the Allee effect - measured by the threshold $\lambda_{\min }-$ on the quasi-distribution of the metapopulation size (see Fig. 4); as expected, the stronger the Allee effect, more concentrated on small sizes the quasi-distribution, because the Allee effect plays

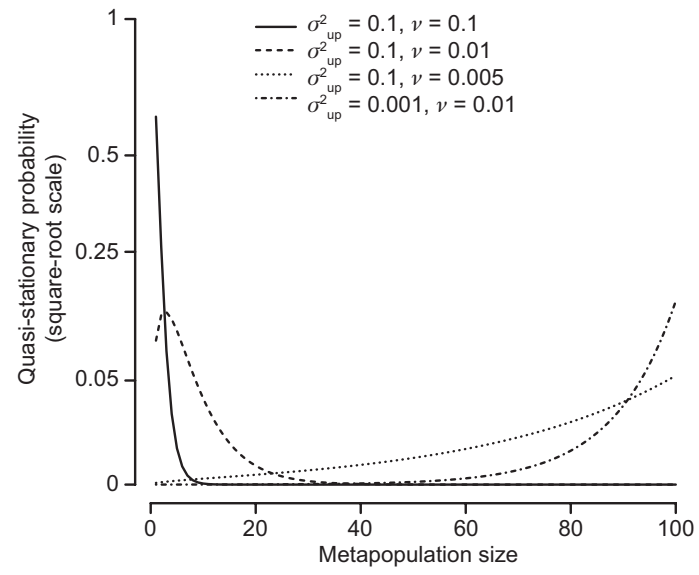

Fig. 5. Quasi-distribution of the metapopulation size for different values of the parameters $\sigma_{\text {up }}^{2}$ and $v$. The other parameters are fixed to $\alpha_{\mathrm{m}}=0.5, \alpha_{\mathrm{d}}=0.5, \eta=1, \lambda_{\text {min }}=$ $0.25, \beta=100, \gamma=1$, and $N^{\max }=100$.

against the survival of SP and the emergence of CP. Interestingly, the change in the shape of the quasi-distribution is relatively abrupt (see Fig. 4): from $\lambda_{\text {min }}=0.13$ to $\lambda_{\text {min }}=0.11$, the quasi-distribution rapidly shifts from preferentially weighting the small sizes to preferentially weighting the large sizes.

Regarding the effect of the strength of the group diffusion - measured by the variance $\sigma_{\text {up }}^{2}$ and the parameter $v-$ on the quasi-distribution of the metapopulation size (see Fig. 5); as expected, stronger the group diffusion, more concentrated on small sizes the quasi-distribution, because the group diffusion plays against the emergence of CP.

Regarding the effect of the size of the migrating groups - measured by the maximum migrating fraction $\alpha_{\mathrm{m}}-$ on the quasi-distribution of the metapopulation size (see Fig. 6); when $\alpha_{\mathrm{m}}$ is small, our metapopulation model can be viewed as a model without group dispersal. Here, in contrast to the two preceding analyses, we observe a non-monotonous effect of $\alpha_{\mathrm{m}}$ : the quasi-distribution is concentrated on small sizes of the metapopulation for large and small values of $\alpha_{\mathrm{m}}$, whereas it is concentrated on larger sizes of the metapopulation for intermediate values of $\alpha_{\mathrm{m}}$. Heuristically, large values of $\alpha_{\mathrm{m}}$ plays against the survival of SP; small values of $\alpha_{\mathrm{m}}$ plays against the emergence of $\mathrm{CP}$; whereas intermediate values of $\alpha_{\mathrm{m}}$ may allow both the survival of SP 


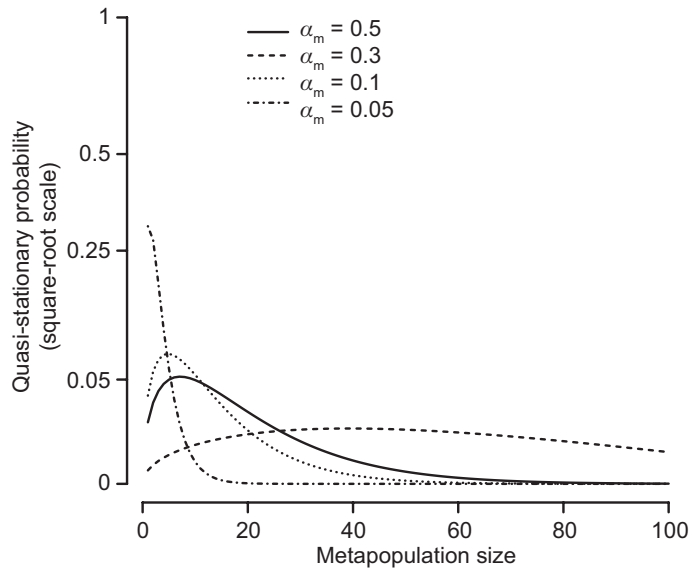

Fig. 6. Quasi-distribution of the metapopulation size for different values of the maximum migrating fraction $\alpha_{\mathrm{m}}$. The other parameters are fixed to $\alpha_{\mathrm{d}}=0.5, \sigma_{\text {up }}^{2}=0.01$, $v=0.001, \eta=1, \lambda_{\min }=0.5, \beta=100, \gamma=1$, and $N^{\max }=$ 100.

and the emergence of CP. The non-monotonous effect of $\alpha_{\mathrm{m}}$ can also be observed on the expected time to extinction: the intermediate value $\alpha_{\mathrm{m}}=$ 0.3 leads on average to longer existence of the metapopulation (see Fig. 7). Finally, it has to be noted that the non-monotonous effect of $\alpha_{\mathrm{m}}$ may not be observed when the other parameters are fixed to other values than those used to create Fig. 6.

\section{Discussion}

Although group dispersal is a phenomenon associated with many species, how metapopulation turnover and size may be influenced by the size of the dispersing group has not been previously investigated. Here, we showed how sensitive metapopulation dynamics are to the size of the dispersing group. Below we discuss our finding in more detail and outline some future key areas of research.

To understand how metapopulation dynamics are affected by the size of the dispersing group, we built a temporal metapopulation model incorporating group dispersal and an Allee effect. Using the framework of Stochastic Patch Occupancy Models (SPOM) initially developed by Ovaskainen and Hanski (2004a), we characterized the metapopulation dynamics as a func-

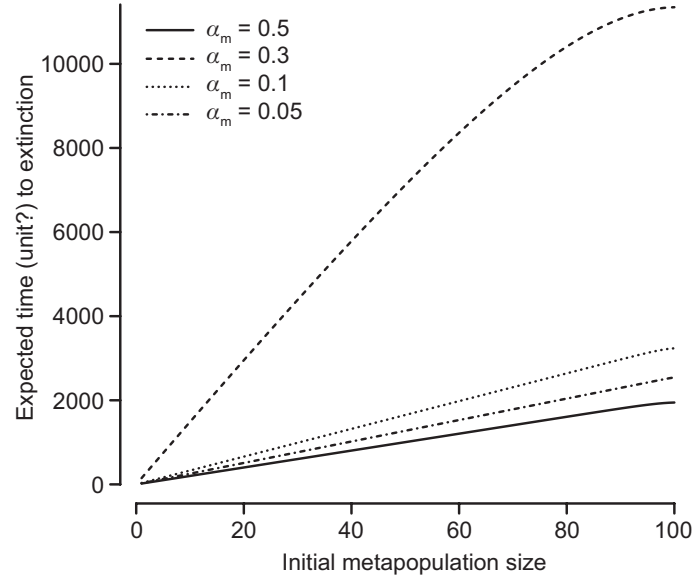

Fig. 7. Expected time to extinction of the metapopulation, conditional on the initial metapopulation size, for different values of the maximum migrating fraction $\alpha_{\mathrm{m}}$. The other parameters are fixed to $\alpha_{\mathrm{d}}=0.5, \sigma_{\text {up }}^{2}=0.01$, $v=0.001, \eta=1, \lambda_{\min }=0.5, \beta=100, \gamma=1$, and $N^{\max }=$ 100.

tion of the Allee effect, group diffusion and group size, which allowed us to identify the conditions under which group dispersal promotes metapopulation dynamics. Heuristically we showed that larger group sizes decrease the probability of survival of the source population while small group sizes decrease the probability of successful establishment. Our results demonstrate that intermediate group sizes may lead to larger and more sustainable metapopulations in the presence of an Allee effect. For any small population, extinction risk is inversely proportional to population size (Lande 1993) due to the effects of demographic stochasticity and Allee effects. Demographic stochasticity is the randomness associated with individuality (Fox 2005), and its impact is most prevalent in small populations because such stochastic variation averages out in larger ones. Allee effects come into play when below a threshold population size, density dependence on population growth goes from negative to positive (e.g., when mates become rare, reproduction cannot happen). Mate availability has also been considered an important factor for obligately outcrossing species, especially those that are also short-lived (Courchamp et al. 1999). In general, Allee effects are expected to play a major role in the formation of a species' geographic range (Keitt et al. 2001). 
For passive airborne dispersal - such as pollen and spore dispersal - the physical environment has been considered crucial for determining the dispersal process (Aylor 1990). In our model we find that when the diffusion of the migrating fraction is large, at least one of the two populations goes extinct. When the migrating fraction is small, the source population survives and the colonizing population vanishes because of the diffusion. Alternatively, when the migrating fraction is large, the source population vanishes and the colonizing population emerges despite the diffusion. Given how variable physical conditions affecting the dispersal process can be, this is expected to generate spatio-temporal variation in real biological systems (Aylor 1990). Although our model has been developed for passively dispersing organisms, such conditiondependency affecting the size of the dispersing group and the distance travelled is also characteristic of actively dispersing species (Rousset 2012), and hence, our results also offer insight for systems supporting active dispersal.

Investigating group dispersal within a metapopulation framework is especially important for pathogens because it elegantly links the key concepts of epidemiology: the location of susceptible hosts (i.e. habitat patches) and transmission between them (i.e. dispersal). Indeed, diseases are frequently aggregated across multiple spatial scales and the local populations are ephemeral as predicted by metapopulation theory (Grenfell \& Harwood 1997, Burdon \& Thrall 2014, Parratt et al. 2016). However, while a large body of literature has explored large-scale geographical patterns of disease (Rambaut et al. 2008, Meentemeyer et al. 2012), and disease dynamics within a metapopulation framework have been analysed in several recent papers (Keeling \& Gilligan 2000, Antonovics 2004, Laine \& Hanski 2006, Soubeyrand et al. 2009, Arino et al. 2012, Jousimo et al. 2014), few empirical studies have focused on the patterns and mechanisms behind disease clusters at small spatial scales. Group dispersal may be one key process driving the observed disease patterns given that pathogen spores are well-known to form clumps in which they travel between hosts (Ingold 1971, Rapilly 1979, Ferrandino \& Aylor 1987, Tack et al.2014). The size of the dispersing unit has been shown to have a direct effect on successful establishment even in the obligate fungal pathogen, Podosphaera plantaginis, that persists as a highly dynamic metapopulation in the Aland Islands: larger spore groups have higher infectivity than solitary spores (Tack et al. 2014). Our results show how sensitive metapopulation dynamics are to the size of the dispersing group, and hence, investigating the epidemiological consequences of group size during pathogen transmission offers an exciting future venue of research.

In our modelling framework, the size of a source population is synchronously decreased by emigration and destruction processes. Therefore, when a group dispersal episode occurs, the source population faces the Allee effect. However, for some taxa, the effect of dispersal on the size of the source population may be negligible. Thus, further investigation should be carried out to see if the non-monotonous effect of $\alpha_{\mathrm{m}}$ (the parameter governing group sizes) on the metapopulation size and the extinction time is modified when dispersal episodes do not immediately affect the source populations. In this case, we should however take into account the limited capacity of source populations to emit groups of dispersers (different assumptions on the regeneration of source populations should be made). Ovaskainen (2017) considered such a case with a different modelling framework and highlighted that an intermediate group size maximizes the colonization rate. Based on this result, we can expect that the non-monotonous influence of $\alpha_{\mathrm{m}}$ might be preserved when dispersal episodes do not immediately affect the source populations. More generally, it would be interesting to investigate under which conditions the non-monotonous influence of group size on metapopulation size and extinction time could be obtained in the absence of an Allee effect. From this perspective, let us evoke the work by Holt and Barfield (2006) and Barfield et al. (2015) on the influence of pathogen shedding on between-host pathogen dynamics. Pathogen shedding is the emission by the infectious host of pathogens throughout the course of infection, and can act as a loss term for the microbial population within the host (similarly, in our model, the migrating and destroyed fractions are subtracted from the source population). Holt and Barfield (2006) have shown that, 
in some circumstances, an intermediate shedding rate leads to the greatest overall growth rate of the pathogen. Thus, we could remove the Allee effect from our model and determine under which conditions on the regeneration of source populations a non-monotonous effect of group size is obtained.

In conclusions, understanding the conditions under which group dispersal promotes metapopulation persistence is vital for accurately accounting for variation in the size of the dispersing group, which can vary significantly both within and between species. Our results confirm that intermediate group sizes are most favourable to metapopulation size and persistence in the presence of an Allee effect. Our results suggest that the size of the dispersing unit may be under direct selection that may vary between continuous and fragmented landscapes. Establishing direct links between the fundamental axes of group size variation and realized (meta)population dynamics offers an exciting future venue of research that is expected to yield key insights into the ecology and evolution of populations occupying spatially structured environments.

\section{Acknowledgements}

We warmly thank R.D. Holt for his comments on the manuscript. SS acknowledges funding from the Plant Health and Environment Division of INRA (project no. 2010-546-1) and A-LL from the Academy of Finland (Center of Excellence in Metapopulation Biology 2015-2017; 284601) and the European Research Council (Independent Starting Grant PATHEVOL; 281517).

\section{References}

Antonovics, J. 2004: Long-term study of a plant-pathogen metapopulation. - In: Hanski, I. \& Gaggiotti, O. (eds.), Ecology, genetics, and evolution of metapopulations, 471-488. Academic Press, Amsterdam, The Netherlands.

Arino, J., Ducrot, A. \& Zongo, P. 2012: A metapopulation model for malaria with transmission-blocking partial immunity in hosts. - Journal of Mathematical Biology 64: 423-448.

Aylor, D. E. 1990: The role of intermittent wind in the dispersal of fungal pathogens. - Annual Review of Phytopathology 28: 73-92.

Barfield, M., Orive, M. E. \& Holt, R. D. 2015: The role of pathogen shedding in linking within- and between-host pathogen dynamics. - Mathematical Biosciences 270: 249-262.

Burdon, J. J. \& Thrall, P. H. 2014: What have we learned from studies of wild plant-pathogen associations? - the dynamic interplay of time, space and life-history. European Journal of Plant Pathology 138: 417-429.

Carlson, A. \& Edenhamn, P. 2000: Extinction dynamics and the regional persistence of a tree frog metapopulation. - Proceedings of the Royal Society B 267: 1311-1313.

Clobert, J., Baguette, M., Benton, T. G. \& Bullock, J. M. 2012: Dispersal ecology and evolution. - Oxford University Press, Oxford.

Courchamp, F., Clutton-Brock, T. \& Grenfell, B. 1999: Inverse density dependence and the Allee effect. Trends in Ecology \& Evolution 14: 405-410.

Darroch, J. N. \& Seneta, E. 1967: On quasi-stationary distributions in absorbing continuous-time finite Markov chains. - Journal of Applied Probability 4: 192-196.

Ferrandino, F. J. \& Aylor, D. E. 1987: Relative abundance and deposition gradients of clusters of Urediniospores of Uromyces phaseoli. - Phytopathology 77: 107-111.

Fox, G. A. 2005: Extinction risk of heterogeneous populations. - Ecology 86: 1191-1198.

Grenfell, B. \& Harwood, J. 1997: (Meta)population dynamics of infectious diseases. - Trends in Ecology \& Evolution 12: 395-399.

Hanski, I. 1999: Metapopulation ecology. — Oxford University Press, Oxford-New York.

Hanski, I. \& Gaggiotti, O. E. 2004: Metapopulation biology: past, present and future. - In: Hanski, I. \& Gaggiotti, O. E. (eds.), Ecology, genetics, and evolution of metapopulations: 3-22. Academic Press, Amsterdam, The Netherlands.

Holt, R. D. \& Barfield, M. 2006: Within-host pathogen dynamics: some ecological and evolutionary consequence of transients, dispersal mode, and within-host spatial heterogeneity. - In: Feng, Z., Dieckmann, U. \& Levin, S. A. (eds.), Disease evolution : models, concepts, and data analyses: 45-66. American Mathematical Society, Providence, RI.

Ingold, C. T. 1971: Fungal spores: their liberation and dispersal. - Clarendon Press, Oxford.

Jousimo, J., Tack, A. J. M., Ovaskainen, O., Mononen, T., Susi, H., Tollenaere, C. \& Laine, A. L. 2014: Disease ecology. Ecological and evolutionary effects of fragmentation on infectious disease dynamics. - Science 344: 1289-1293.

Keeling, M. J. \& Gilligan, C. A. 2000: Metapopulation dynamics of bubonic plague. - Nature 407: 903-906.

Keitt, T. H., Lewis, M. A. \& Holt, R. D. 2001: Allee effects, invasion pinning, and species' borders. - American Naturalist 157: 203-216.

Lahaye, W. S., Gutierrez, R. J. \& Akcakaya, H. R. 1994: Spotted owl metapopulation dynamics in southern California. - Journal of Animal Ecology 63: 775-785.

Laine, A. L. \& Hanski, I. 2006: Large-scale spatial dynamics of a specialist plant pathogen in a fragmented landscape. - Journal of Ecology 94: 217-226.

Lande, R. 1993: Risks of population extinction from demo- 
graphic and environmental stochasticity and random catastrophes. - American Naturalist 142: 911-927.

Levins, R. 1969: Some demographic and genetic consequences of environmental heterogeneity for biological control. - Bulletin for the Entomological Society of America 15: 237-240.

Levins, R. 1970: Extinction. - In: Gerstenhaber, M. (ed.) Some mathematical questions in biology. Lectures on mathematics in the life sciences: 75-107. American Mathematical Society, Providence, RI.

Matthysen, E. 2012: Multicausality of dispersal: a review. In: Clobert, J., Baguette, M., Benton, T. G. \& Bullock, J. M. (eds.), Dispersal ecology and evolution, 1st ed.: 3-18. Oxford University Press, Oxford.

Meentemeyer, R. K., Haas, S. E. \& Vaclavik, T. 2012: Landscape epidemiology of emerging infectious diseases in natural and human-altered ecosystems. - Annual Review of Phytopathology 50: 379-402.

Meerson, B. \& Ovaskainen, O. 2013: Immigration-extinction dynamics of stochastic populations. - Physical Review E 88,012124, doi:10.1103/PhysRevE.88.012124.

Nathan, R., Klein, E., Robledo-Arnuncio, J. J. \& Revilla, E. 2012: Dispersal kernels: review. - In: Clobert, J., Baguette, M., Benton, T. G. \& Bullock, J. M. (eds.), Dispersal ecology and evolution, 1st ed.: 187-210. Oxford University Press, Oxford.

Ouborg, N. J. 1993: Isolation, population-size and extinction: the classical and metapopulation approaches applied to vascular plants along the Dutch Rhine-system. - Oikos 66: 298-308.

Ovaskainen, O. 2017: The interplay between immigration and local population dynamics in metapopulations. Annales Zoologici Fennici 54: 113-121.

Ovaskainen, O. \& Hanski, I. 2004a: From individual behavior to metapopulation dynamics: Unifying the patchy population and classic metapopulation models. - American Naturalist 164: 364-377.

Ovaskainen, O. \& Hanski, I. 2004b: Metapopulation dynamics in highly fragmented landscapes. - In: Hanski, I. \& Gaggiotti, O. E. (eds.), Ecology, genetics, and evolution of metapopulations: 73-103. Academic Press, Amsterdam, The Netherlands.

Parratt, S. R., Numminen, E. \& Laine, A. L. 2016: Infec- tious disease dynamics in heterogeneous landscapes. Annual Review of Ecology, Evolution, and Systematics 47: 283-306.

Rambaut, A., Pybus, O. G., Nelson, M. I., Viboud, C., Taubenberger, J. K. \& Holmes, E. C. 2008: The genomic and epidemiological dynamics of human influenza A virus. - Nature 453: 615-612.

Rapilly, F. 1979: Yellow rust epidemiology. - Annual Review of Phytopathology 17: 59-73.

Ronce, O. 2007: How does it feel to be like a rolling stone? Ten questions about dispersal evolution. - Annual Review of Ecology Evolution and Systematics 38: 231-253.

Rousset, F. 2012: Demographic consequences of the selective forces controlling density-dependent dispersal. In: Clobert, J., Baguette, M., Benton, T. G. \& Bullock, J. M. (eds.), Dispersal ecology and evolution, 1st ed.: 266-279. Oxford University Press, Oxford.

Soubeyrand, S., Mrkvicka, T. \& Penttinen, A. 2014: A nonstationary cylinder-based model describing group dispersal in a fragmented habitat. - Stochastic Models 30: $48-67$.

Soubeyrand, S., Laine, A. L., Hanski, I. \& Penttinen, A. 2009: Spatiotemporal structure of host-pathogen interactions in a metapopulation. - American Naturalist 174: 308-320.

Soubeyrand, S., Roques, L., Coville, J. \& Fayard, J. 2011: Patchy patterns due to group dispersal. - Journal of Theoretical Biology 271: 87-99.

Soubeyrand, S., Sache, I., Hamelin, F. \& Klein, E. K. 2015: Evolution of dispersal in asexual populations: to be independent, clumped or grouped? - Evolutionary Ecology 29: 947-963.

Starrfelt, J. \& Kokko, H. 2012: The multicausal nature of dispersal. - In: Clobert, J., Baguette, M., Benton, T. G. \& Bullock, J. M. (eds.), Dispersal ecology and evolution, 1st ed.: 19-28. Oxford University Press, Oxford.

Tack, A. J. M., Hakala, J., Petaja, T., Kulmala, M. \& Laine, A. L. 2014: Genotype and spatial structure shape pathogen dispersal and disease dynamics at small spatial scales. - Ecology 95: 703-714.

Travis, J. M. J. \& Dytham, C. 2002: Dispersal evolution during invasions. - Evolutionary Ecology Research 4: 1119-1129. 


\section{Appendix}

\section{Transition probabilities}

Increment +1

Let us define the following quantities:

$$
\begin{aligned}
& A_{0}(r)=2 \pi\left(\sigma_{\mathrm{up}}^{2}+v r^{\eta}\right) \lambda_{\min }, \\
& B_{1}=\min \left\{\alpha_{\mathrm{m}}, 1-\lambda_{\min }\right), \\
& A_{1}(r)=\min \left\{B_{1}, \max \left\{A_{0}(r), 1-\lambda_{\min }-\alpha_{\mathrm{d}}\right\}\right\}, \\
& B_{2}=\min \left\{\alpha_{\mathrm{m}}, 1-\lambda_{\min }-\alpha_{\mathrm{d}}\right\}, \text { and } \\
& A_{2}(r)=\min \left\{B_{2}, A_{0}(r)\right\} .
\end{aligned}
$$

When $1 \leq N_{i} \leq N^{\max }$,

$$
\begin{aligned}
& p_{+1}=\mathbb{P}\left(N_{i+1}=N_{i}+1\right) \\
& =\int_{\mathrm{R}_{+} \times[0,1]^{1}} \mathbb{1}\left(1-\lambda_{\mathrm{m}}-\lambda_{\mathrm{d}} \geq \lambda_{\text {min }}\right) \mathbb{1}\left(\lambda_{\mathrm{m}} \geq A_{0}(r)\right) \\
& \times \frac{1}{\alpha_{\mathrm{m}}} \mathbb{1}\left(0 \leq \lambda_{\mathrm{m}} \leq \alpha_{\mathrm{m}}\right) \frac{1}{\alpha_{\mathrm{d}}} \mathbb{1}\left(0 \leq \lambda_{\mathrm{d}} \leq \alpha_{\mathrm{d}}\right) g(r) d \lambda_{\mathrm{m}} d \lambda_{\mathrm{d}} d r \\
& =\int_{\mathrm{R}_{+} \times[0,1]} \mathbb{1}\left(\lambda_{\mathrm{m}} \geq A_{0}(r)\right) \frac{g(r)}{\alpha_{\mathrm{m}} \alpha_{\mathrm{d}}} \mathbb{1}\left(0 \leq \lambda_{\mathrm{m}} \leq \alpha_{\mathrm{m}}\right) \\
& \times \min \left\{\alpha_{\mathrm{d}}, 1-\lambda_{\min }-\lambda_{\mathrm{m}}\right\} \mathbb{1}\left(\lambda_{\mathrm{m}}<1-\lambda_{\min }\right) d \lambda_{\mathrm{m}} d r \\
& =\int_{\mathrm{R}_{+}} \frac{g(r)}{\alpha_{\mathrm{m}} \alpha_{\mathrm{d}}} \int_{0}^{1} \mathbb{1}\left(\lambda_{\mathrm{m}}<1-\lambda_{\text {min }}\right) \mathbb{1}\left(0 \leq \lambda_{\mathrm{m}} \leq \alpha_{\mathrm{m}}\right) \mathbb{1}\left(\lambda_{\mathrm{m}} \geq A_{0}(r)\right) \\
& \times\left[\left(1-\lambda_{\text {min }}-\lambda_{\mathrm{m}}\right) \mathbb{1}\left(1-\lambda_{\text {min }}-\lambda_{\mathrm{m}} \leq \alpha_{\mathrm{d}}\right)+\alpha_{\mathrm{d}} \mathbb{1}\left(1-\lambda_{\text {min }}-\lambda_{\mathrm{m}}>\alpha_{\mathrm{d}}\right)\right] d \lambda_{\mathrm{m}} d r \\
& =\int_{\mathrm{R}_{+}} \frac{g(r)}{\alpha_{\mathrm{m}} \alpha_{\mathrm{d}}} \int_{A_{1}(r)}^{B_{1}}\left(1-\lambda_{\min }-\lambda_{\mathrm{m}}\right) d \lambda_{\mathrm{m}} d r+\int_{\mathrm{R}_{+}} \frac{g(r)}{\alpha_{\mathrm{m}} \alpha_{\mathrm{d}}} \int_{A_{2}(r)}^{B_{2}} \alpha_{\mathrm{d}} d \lambda_{\mathrm{m}} d r
\end{aligned}
$$

We now compute the first integral in the last line of Eq. A1.

If $1-\lambda_{\text {min }}-\alpha_{\mathrm{d}} \geq B_{1}$, then, for all $r \geq 0, A_{1}(r)=B_{1}$ and $\int_{A_{1}(r)}^{B_{1}}\left(1-\lambda_{\min }-\lambda_{\mathrm{m}}\right) d \lambda_{\mathrm{m}}=0$.

If $1-\lambda_{\min }-\alpha_{\mathrm{d}}<B_{1}$, then

- for all $r \in\left[0, R_{1}\right]$, with $R_{1}=\left\{\frac{1}{v}\left(\frac{1-\lambda_{\min }-\alpha_{d}}{2 \pi \lambda_{\min }}-\sigma_{\text {up }}^{2}\right)\right\}^{1 / \eta}, A_{0}(r) \leq 1-\lambda_{\min }-\alpha_{\mathrm{d}}$ and, consequently, $A_{1}(r)=1-\lambda_{\min }-\alpha_{\mathrm{d}}$

- for all $r \in\left(R_{1}, R_{2}\right]$, with $R_{2}=\left\{\frac{1}{v}\left(\frac{B_{1}}{2 \pi \lambda_{\text {min }}}-\sigma_{\text {up }}^{2}\right)\right\}^{1 / \eta}, 1-\lambda_{\text {min }}-\alpha_{\mathrm{d}}<A_{0}(r) \leq B_{1}$ and, consequently, $A_{1}(r)=A_{0}(r)=2 \pi\left(\sigma_{\text {up }}^{2} \pm v r^{\eta}\right) \lambda_{\text {min }} ;$

- for all $r>R_{2}, A_{1}(r)=B_{1}$ and $\int_{A_{1}(r)}^{B_{1}}\left(1-\lambda_{\min }-\lambda_{\mathrm{m}}\right) d \lambda_{\mathrm{m}}=0$. 
Therefore,

$$
\begin{aligned}
& \int_{\mathrm{R}_{+}} \frac{g(r)}{\alpha_{\mathrm{m}} \alpha_{\mathrm{d}}} \int_{A_{1}(r)}^{B_{1}}\left(1-\lambda_{\min }-\lambda_{\mathrm{m}}\right) d \lambda_{\mathrm{m}} d r \\
& =\mathbb{1}\left(1-\lambda_{\min }-\alpha_{\mathrm{d}}<B_{1}\right)\left(\int_{0}^{R_{1}} \frac{g(r)}{\alpha_{\mathrm{m}} \alpha_{\mathrm{d}}} \int_{1-\lambda_{\min }-\alpha_{\mathrm{d}}}^{B_{1}}\left(1-\lambda_{\min }-\lambda_{\mathrm{m}}\right) d \lambda_{\mathrm{m}} d r\right. \\
& \left.+\int_{R_{1}}^{R_{2}} \frac{g(r)}{\alpha_{\mathrm{m}} \alpha_{\mathrm{d}}} \int_{2 \pi\left(\sigma_{\mathrm{up}}^{2}+v r^{\eta}\right) \lambda_{\min }}^{B_{1}}\left(1-\lambda_{\min }-\lambda_{\mathrm{m}}\right) d \lambda_{\mathrm{m}} d r\right) \\
& =\mathbb{1}\left(1-\lambda_{\min }-\alpha_{\mathrm{d}}<B_{1}\right)\left(C_{1} \int_{0}^{R_{1}} g(r) d r+C_{2} \int_{R_{1}}^{R_{2}} g(r) d r+C_{3} \int_{R_{1}}^{R_{2}} r^{\eta} g(r) d r+C_{4} \int_{R_{1}}^{R_{2}} r^{2 \eta} g(r) d r\right) \text {, } \\
& =\mathbb{1}\left(1-\lambda_{\min }-\alpha_{\mathrm{d}}<B_{1}\right)\left[C_{1} F\left(R_{1} ; \frac{1}{\gamma}, \beta\right)+C_{2}\left\{F\left(R_{2} ; \frac{1}{\gamma}, \beta\right)-F\left(R_{1} ; \frac{1}{\gamma}, \beta\right)\right\}\right. \\
& +C_{3} Z(\eta)\left\{F\left(R_{2} ; \frac{\eta+1}{\gamma}, \beta\right)-F\left(R_{1} ; \frac{\eta+1}{\gamma}, \beta\right)\right\} \\
& \left.+C_{4} Z(2 \eta)\left\{F\left(R_{2} ; \frac{2 \eta+1}{\gamma}, \beta\right)-F\left(R_{1} ; \frac{2 \eta+1}{\gamma}, \beta\right)\right\}\right]
\end{aligned}
$$

where

$$
\begin{aligned}
& C_{1}=\frac{1}{\alpha_{\mathrm{m}} \alpha_{\mathrm{d}}}\left(\left(1-\lambda_{\text {min }}\right)\left\{B_{1}-\left(1-\lambda_{\text {min }}-\alpha_{\mathrm{d}}\right)\right\}-\frac{1}{2}\left\{B_{1}^{2}-\left(1-\lambda_{\text {min }}-\alpha_{\mathrm{d}}\right)^{2}\right\}\right) \\
& C_{2}=\frac{1}{\alpha_{\mathrm{m}} \alpha_{\mathrm{d}}}\left(\left(1-\lambda_{\min }\right)\left\{B_{1}-2 \pi \sigma_{\mathrm{up}}^{2} \lambda_{\min }\right\}-\frac{1}{2}\left\{B_{1}^{2}-\left(2 \pi \sigma_{\mathrm{up}}^{2} \lambda_{\min }\right)^{2}\right\}\right) \\
& C_{3}=\frac{1}{\alpha_{\mathrm{m}} \alpha_{\mathrm{d}}}\left(-\left(1-\lambda_{\min }\right) 2 \pi v \lambda_{\text {min }}+\left(2 \pi \lambda_{\text {min }}\right)^{2} \sigma_{\mathrm{up}}^{2} \nu\right) \\
& C_{4}=\frac{1}{2 \alpha_{\mathrm{m}} \alpha_{\mathrm{d}}}\left(2 \pi v \lambda_{\min }\right)^{2} \\
& Z(u)=\frac{\beta^{u / \gamma} \Gamma\left(\frac{u+1}{\gamma}\right)}{\Gamma\left(\frac{1}{\gamma}\right)},
\end{aligned}
$$

and $F(\bullet ; a, s)$ is the cumulative distribution function of a random variable drawn from the gamma distribution with shape parameter $a$ and scale parameter $s$.

We now compute the second integral in the last line of Eq. A1:

- for all $r \in\left[0, R_{3}\right]$, with $R_{3}=\left\{\frac{1}{v}\left(\frac{B_{2}}{2 \pi \lambda_{\text {min }}}-\sigma_{\text {up }}^{2}\right)\right\}^{1 / \eta}, A_{0}(r) \leq B_{2}$ and, consequently, $A_{2}(r)=A_{0}(r)=$
$2 \pi\left(\sigma_{\text {up }}^{2} \pm v r^{\eta}\right) \lambda_{\text {min }} ;$

- for all $r>R_{3}, A_{2}(r) \leq B_{2}$ and $\int_{A_{2}(r)}^{B_{2}} \alpha_{\mathrm{d}} d \lambda_{\mathrm{m}}=0$. 
ANN.ZOOL.FENNICI Vol.54 • How group dispersal shapes metapopulations

137

Therefore,

$$
\begin{aligned}
\int_{\mathbb{R}_{+}} \frac{g(r)}{\alpha_{\mathrm{m}} \alpha_{\mathrm{d}}} \int_{A_{2}(r)}^{B_{2}} \alpha_{\mathrm{d}} d \lambda_{\mathrm{m}} d r & =\int_{0}^{R_{3}} \frac{g(r)}{\alpha_{\mathrm{m}} \alpha_{\mathrm{d}}} \int_{2 \pi\left(\sigma_{\mathrm{tp}^{2}}^{2}+v r^{\eta}\right) \lambda_{\min }}^{B_{2}} \alpha_{\mathrm{d}} d \lambda_{\mathrm{m}} d r \\
& =C_{5} \int_{0}^{R_{3}} g(r) d r+C_{6} \int_{0}^{R_{3}} r^{\eta} g(r) d r \\
& =C_{5} F\left(R_{3} ; \frac{1}{\gamma}, \beta\right)+C_{6} Z(\eta) F\left(R_{3} ; \frac{\eta+1}{\gamma}, \beta\right)
\end{aligned}
$$

where $C_{5}=\frac{1}{\alpha_{\mathrm{m}}}\left(B_{2}-2 \pi \sigma_{\text {up }}^{2} \lambda_{\min }\right)$ and $C_{6}=-\frac{2 \pi v \lambda_{\min }}{\alpha_{\mathrm{m}}}$.

Consequently,

$$
\begin{aligned}
p_{+1}=\mathbb{1}\left(1-\lambda_{\min }-\alpha_{\mathrm{d}}<B_{1}\right)[ & C_{1} F\left(R_{1} ; \frac{1}{\gamma}, \beta\right)+C_{2}\left\{F\left(R_{2} ; \frac{1}{\gamma}, \beta\right)-F\left(R_{1} ; \frac{1}{\gamma}, \beta\right)\right\} \\
& +C_{3} Z(\eta)\left\{F\left(R_{2} ; \frac{\eta+1}{\gamma}, \beta\right)-F\left(R_{1} ; \frac{\eta+1}{\gamma}, \beta\right)\right\} \\
& \left.+C_{4} Z(2 \eta)\left\{F\left(R_{2} ; \frac{2 \eta+1}{\gamma}, \beta\right)-F\left(R_{1} ; \frac{2 \eta+1}{\gamma}, \beta\right)\right\}\right] \\
& +C_{5} F\left(R_{3} ; \frac{1}{\gamma}, \beta\right)+C_{6} Z(\eta) F\left(R_{3} ; \frac{\eta+1}{\gamma}, \beta\right)
\end{aligned}
$$

Increment -1

Let us define the following quantities:

$$
\begin{aligned}
& A_{3}=1-\lambda_{\text {min }}-\alpha_{\mathrm{d}}, \\
& B_{3}(r)=\max \left\{A_{3}, \min \left\{A_{0}(r), \alpha_{\mathrm{m}}\right\}\right\} .
\end{aligned}
$$

When $1 \leq N_{i} \leq N^{\max }$,

$$
\begin{aligned}
& p_{-1}= P\left(N_{i+1}=N_{i}-1\right) \\
&= \int_{\mathrm{R}_{+} \times[0,1]^{2}} \mathbb{1}\left(1-\lambda_{\mathrm{m}}-\lambda_{\mathrm{d}}<\lambda_{\text {min }}\right) \mathbb{1}\left(\lambda_{\mathrm{m}}<A_{0}(r)\right) \\
& \quad \times \frac{1}{\alpha_{\mathrm{m}}} \mathbb{1}\left(0 \leq \lambda_{\mathrm{m}} \leq \alpha_{\mathrm{m}}\right) \frac{1}{\alpha_{\mathrm{d}}} \mathbb{1}\left(0 \leq \lambda_{\mathrm{d}} \leq \alpha_{\mathrm{d}}\right) g(r) d \lambda_{\mathrm{m}} d \lambda_{\mathrm{d}} d r \\
&= \int_{\mathrm{R}_{+} \times[0,1]} \mathbb{1}\left(\lambda_{\mathrm{m}}<A_{0}(r)\right) \frac{g(r)}{\alpha_{\mathrm{m}} \alpha_{d}} \mathbb{1}\left(0 \leq \lambda_{\mathrm{m}} \leq \alpha_{\mathrm{m}}\right) \\
& \quad \times\left\{\alpha_{\mathrm{d}}-\left(1-\lambda_{\text {min }}-\lambda_{\mathrm{m}}\right)\right\} \mathbb{1}\left(\alpha_{d}>1-\lambda_{\text {min }}-\lambda_{\mathrm{m}}\right) d \lambda_{\mathrm{m}} d r \\
&=\int_{\mathrm{R}_{+}} \frac{g(r)}{\alpha_{\mathrm{m}} \alpha_{\mathrm{d}}} \int_{0}^{1}\left\{\alpha_{\mathrm{d}}-\left(1-\lambda_{\min }-\lambda_{\mathrm{m}}\right)\right\} \mathbb{1}\left(\lambda_{\mathrm{m}}<A_{0}(r)\right) \mathbb{1}\left(0 \leq \lambda_{\mathrm{m}} \leq \alpha_{\mathrm{m}}\right) \\
& \quad \times \mathbb{1}\left(\lambda_{\mathrm{m}}>1-\lambda_{\min }-\alpha_{\mathrm{d}}\right) d \lambda_{\mathrm{m}} d r \\
&=\int_{\mathrm{R}_{+}} \frac{g(r)}{\alpha_{\mathrm{m}} \alpha_{\mathrm{d}}} \int_{A_{3}}^{B_{3}(r)}\left(\lambda_{\mathrm{m}}-A_{3}\right) d \lambda_{\mathrm{m}} d r
\end{aligned}
$$


If $\alpha_{\mathrm{m}}<A_{3}$, then, for all $r \geq 0, B_{3}(r)=A_{3}$ and $\int_{A_{3}}^{B_{3}(r)}\left(\lambda_{\mathrm{m}}-A_{3}\right) d \lambda_{\mathrm{m}}=0$.

If $\alpha_{\mathrm{m}} \geq A_{3}$, then

- for all $r \in\left[0, R_{4}\right]$, with $R_{4}=\left\{\frac{1}{v}\left(\frac{A_{3}}{2 \pi \lambda_{\text {min }}}-\sigma_{\text {up }}^{2}\right)\right\}^{1 / \eta}, A_{0}(r) \leq A_{3}, B_{3}(r)=A_{3}$ and, consequently, $\int_{A_{3}}^{B_{3}(r)}\left(\lambda_{\mathrm{m}}-A_{3}\right) d \lambda_{\mathrm{m}}=0$

- for all $r \in\left(R_{4}, R_{5}\right.$ ], with $R_{5}=\left\{\frac{1}{v}\left(\frac{\alpha_{\mathrm{m}}}{2 \pi \lambda_{\text {min }}}-\sigma_{\text {up }}^{2}\right)\right\}^{1 / \eta}, A_{3}<A_{0}(r) \leq \alpha_{\mathrm{m}}$ and, consequently, $B_{3}(r)=$ $A_{0}(r)=2 \pi\left(\sigma_{\text {up }}^{2}+v r^{\eta}\right) \lambda_{\text {min }}$

- for all $r>R_{5}, A_{0}(r)>\alpha_{\mathrm{m}}$ and, consequently, $B_{3}(r)=\alpha_{\mathrm{m}}$.

Therefore,

$$
\begin{aligned}
& p_{-1}=\mathbb{1}\left(\alpha_{\mathrm{m}} \geq A_{3}\right)\left(\int_{R_{4}}^{R_{5}} \frac{g(r)}{\alpha_{\mathrm{m}} \alpha_{\mathrm{d}}} \int_{A_{3}}^{2 \pi\left(\sigma_{\text {vp }}^{2}+v r^{\eta}\right) \lambda_{\min }}\left(\lambda_{\mathrm{m}}-A_{3}\right) d \lambda_{\mathrm{m}} d r\right. \\
&\left.\quad+\int_{R_{5}}^{+\infty} \frac{g(r)}{\alpha_{\mathrm{m}} \alpha_{\mathrm{d}}} \int_{A_{3}}^{\alpha_{\mathrm{m}}}\left(\lambda_{\mathrm{m}}-A_{3}\right) d \lambda_{\mathrm{m}} d r\right) \\
&=\mathbb{1}\left(\alpha_{\mathrm{m}} \geq A_{3}\right)\left(C_{7} \int_{R_{4}}^{R_{5}} g(r) d r+C_{8} \int_{R_{4}}^{R_{5}} r^{\eta} g(r) d r+C_{9} \int_{R_{4}}^{R_{5}} r^{2 \eta} g(r) d r+C_{10} \int_{R_{5}}^{+\infty} g(r) d r\right) \\
&=\mathbb{1}\left(\alpha_{\mathrm{m}} \geq A_{3}\right)\left[C_{7}\left\{F\left(R_{5} ; \frac{1}{\gamma}, \beta\right)-F\left(R_{4} ; \frac{1}{\gamma}, \beta\right)\right\}\right. \\
&+C_{8} Z(\eta)\left\{F\left(R_{5} ; \frac{\eta+1}{\gamma}, \beta\right)-F\left(R_{4} ; \frac{\eta+1}{\gamma}, \beta\right)\right\} \\
&+ C_{9} Z(2 \eta)\left\{F\left(R_{5} ; \frac{2 \eta+1}{\gamma}, \beta\right)-F\left(R_{4} ; \frac{2 \eta+1}{\gamma}, \beta\right)\right\} \\
&\left.+C_{10}\left\{1-F\left(R_{5} ; \frac{1}{\gamma}, \beta\right)\right\}\right]
\end{aligned}
$$

where

$$
\begin{aligned}
& C_{7}=\frac{1}{2 \alpha_{\mathrm{m}} \alpha_{\mathrm{d}}}\left(2 \pi \sigma_{\mathrm{up}}^{2} \lambda_{\text {min }}-A_{3}\right)^{2} \\
& C_{8}=\frac{1}{\alpha_{\mathrm{m}} \alpha_{\mathrm{d}}} 2 \pi v \lambda_{\text {min }}\left(2 \pi \sigma_{\mathrm{up}}^{2} \lambda_{\min }-A_{3}\right) \\
& C_{9}=\frac{\left(2 \pi v \lambda_{\text {min }}\right)^{2}}{2 \alpha_{\mathrm{m}} \alpha_{\mathrm{d}}} \\
& C_{10}=\frac{1}{2 \alpha_{\mathrm{m}} \alpha_{\mathrm{d}}}\left(\alpha_{\mathrm{m}}-A_{3}\right)^{2}
\end{aligned}
$$

